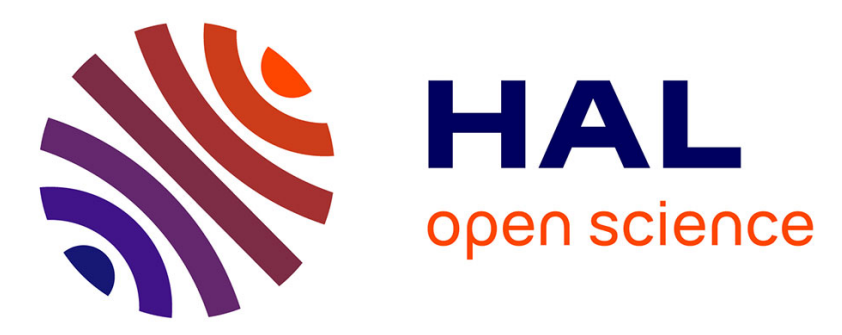

\title{
A robust PI passivity-based control of nonlinear systems and its application to temperature regulation
}

Stanislav Aranovskiy, Romeo Ortega, Rafael Cisneros

\section{To cite this version:}

Stanislav Aranovskiy, Romeo Ortega, Rafael Cisneros. A robust PI passivity-based control of nonlinear systems and its application to temperature regulation. International Journal of Robust and Nonlinear Control, 2016, 26 (10), pp.2216 - 2231. 10.1002/rnc.3404 . hal-01612254

\section{HAL Id: hal-01612254 \\ https://hal-centralesupelec.archives-ouvertes.fr/hal-01612254}

Submitted on 17 Jun 2020

HAL is a multi-disciplinary open access archive for the deposit and dissemination of scientific research documents, whether they are published or not. The documents may come from teaching and research institutions in France or abroad, or from public or private research centers.
L'archive ouverte pluridisciplinaire HAL, est destinée au dépôt et à la diffusion de documents scientifiques de niveau recherche, publiés ou non, émanant des établissements d'enseignement et de recherche français ou étrangers, des laboratoires publics ou privés. 


\title{
A Robust PI Passivity-based Control of Nonlinear Systems and its Application to Temperature Regulation
}

\author{
S. Aranovskiy ${ }^{1,2}$, R. Ortega ${ }^{3}$ and R. Cisneros ${ }^{3 *}$ \\ ${ }^{1}$ School of Automation, Hangzhou Dianzi University, Xiasha Higher Education Zone, Hangzhou, Zhejiang 310018, \\ P.R.China \\ ${ }^{2}$ Department of Control Systems and Informatics, ITMO University, Kronverksiky 49, Saint-Petersburg, Russia \\ ${ }^{3}$ LSS-Supelec, 3 rue Joliot-Curie, 91192 Gif-sur-Yvette, France
}

\begin{abstract}
SUMMARY
This paper deals with the problem of control of partially known nonlinear systems, which have an openloop stable equilibrium, but we would like to add a PI controller to regulate its behavior around another operating point. Our main contribution is the identification of a class of systems for which a globally stable PI can be designed knowing only the systems input matrix and measuring only the actuated coordinates. The construction of the PI is done invoking passivity theory. The difficulties encountered in the design of adaptive PI controllers with the existing theoretical tools are also discussed. As an illustration of the theory we consider a class of thermal processes. Copyright (C)0000 John Wiley \& Sons, Ltd.
\end{abstract}

Received ...

KEY WORDS: Robust PI control; Passivity-based control ; PH systems; Temperature regulation.

\section{INTRODUCTION}

In many practical applications the plant to be controlled has an open-loop stable equilibrium, e.g., at the origin, and we would like to add a controller to regulate its behavior around another operating point. In the case of linear systems the dynamics remains invariant under coordinate shifts, therefore this task can be easily accomplished using the incremental model of the plant. Unfortunately, this is not the case for nonlinear systems, for which there is no obvious advantage of working with the incremental model.

Another common requirement in applications is the use of standard PI controllers, which overwhelmingly dominate the industrial market. Although commissioning a PI to operate around

\footnotetext{
$\overline{{ }^{*} \text { Correspondence }}$ to: R. Cisneros. LSS-Supelec, 3 rue Joliot-Curie, 91192 Gif-sur-Yvette, France. Email:rafael.cisneros@1ss.supelec.fr

Contract/grant sponsor: This article is supported by the Ministry of Education and Science of Russian Federation (project 14.Z50.31.0031) and by Government of Russian Federation (Grant 074-U01)
} 
a single operating point is relatively easy, the performance will be below par in wide operating regimes, which is the scenario in modern high-performance applications. To overcome this drawback the current practice is to re-tune the gains of the PI controllers based on a linear model of the plant evaluated at various operating points, a procedure known as gain-scheduling. There are several disadvantages of gain-scheduling including the need to switch (or interpolate) the controller gains and the non-trivial definition of the regions in the plants state space where the switching takes place-both problems are exacerbated if the dynamics of the plant is highly nonlinear. Another common commissioning procedure is to use auto-tuners, that heavily rely on the availability of a "good" linear approximation of the plant dynamics. To avoid the need to rely on linearization it is necessary to develop a procedure to design robust PI controllers for nonlinear systems with uncertain parameters.

Motivated by the discussion above in this paper we identify a class of (input affine) nonlinear systems for which it is possible to design a PI controller with the following features.

F1 Regulation of the closed-loop system around the desired (non-zero) operating point should be guaranteed.

F2 The PI controller should be robust, in the sense that reduced knowledge of the system parameters is required.

F3 To simplify the controllers commissioning, a well defined admissible range of variation for the PI proportional and integral gains, preserving closed-loop stability, should be provided.

We propose the construction of a PI controller with the features F1-F3 for plants with unknown dynamics verifying the following assumptions.

A1 The open-loop system is unknown but has a stable equilibrium at the origin.

A2 The desired equilibrium belongs to the assignable set and admits a convex Lyapunov function.

A3 The Lyapunov function is the sum of two functions, depending on the un-actuated and actuated coordinates, respectively. The first function is unknown while the second one is separable and linearly parameterized in terms of some unknown parameters.

A4 The input matrix is constant, known and has $n-m$ zero rows, where $n$ and $m$ are the dimensions of the state and input vectors, respectively.

As indicated in the article's title we exploit the fundamental property of passivity to design the PI, which will be referred in the sequel as PI Passivity-based Control (PI-PBC). The first step in the design is to, relying on A1 above, invoke the celebrated theorem of Hill and Moylan [1] to identify a suitable passive output for the system, with storage function the Lyapunov function of the open-loop system. Since our interest is the regulation of non-zero equilibria, we then use the results of [2] to create a new passive output for the incremental model with a storage function that has a minimum at the desired equilibrium. As shown in [2], feeding back the passive output through a PI controller ensures stability of the desired equilibrium for all positive definite PI gains. It is important to underscore that, since the passivity property has been established for the incremental model, the equilibrium can also be stabilized setting the control input equal to the (constant) value that assigns the equilibrium, say $u^{*}$, which is univocally defined. However, this open-loop control will, obviously, be non-robust. In the robustness context of the present paper neither the plant dynamics nor the Lyapunov function are known and, consequently, we cannot compute neither the 
passive output nor $u^{*}$. It is at this point that we invoke A3 and A4 above to prove that, under these assumptions, it is possible to define suitable proportional and integral gains that make the PI-PBC implementable and, consequently, guarantee stability of the equilibrium. Another important feature of the proposed PI-PBC is that it requires only partial measurement of the state, namely, only the $m$ state variables associated to the non-zero rows of the input matrix, referred in the sequel as actuated coordinates. In this way, our approach is oriented towards a characterization of a class of systems that can be regulated by means of the PI-PBC with a minimum knowledge of the system parameters

Several practical applications of PI-PBC have been reported in the literature. This include, RLC circuits [3], power converters [4], fuel cells [5], electric drives [6] and mechanical systems [7]. In [8] a procedure to add an integral action to a non-passive output for a class of port-Hamiltonian systems was first proposed, and later extended in [9], [10]. To the best of our knowledge, the present paper is the first attempt to design PI-PBCs with guaranteed stability properties for systems with partially known dynamics.

A natural question that arises at this point is the incorporation of adaptation in the design of the PI (or PID). In the power converter application of [4] a parameter that enters in the definition of the passive output, i.e., the load resistance, is adaptively identified-however, all other parameters are assumed to be known. In the interesting paper [11] it is shown that it is possible to adaptively estimate $u^{*}$ for a general nonlinear system with scalar input, keeping the estimate in a known interval, provided the passive output is known. In spite of a large number of publications the problem of designing a provably stable adaptive PID for systems with unknown parameters remains, as far as we know, open. The difficulty of this task was identified already in 1984 in [12]. As is well-known [13], the stability of indirect adaptive methods relies on parameter convergence that, in its turn, requires persistency of excitation-a property that is not satisfied in the regulation tasks where PI control is effective. On the other hand, the application of direct methods is stymied by the absence of a suitable parameterization of this structure-constrained controller. For the PI-PBC studied in this paper the main difficulty is the need to estimate two objects, that appear multiplicatively in the Lyapunov analysis: the passive output and the ideal control signal $u^{*}$. This point is further elaborated in Subsection 5.2.

The remaining of the paper is organized as follows. Section 2 presents the problem formulation. To streamline the presentation of the main result, which is given in Section 4, some preliminary lemmata are given in Section 3. In Section 5 we discuss the reasons that stymie the use of adaptation and the inability to state a robustness result based on continuity and approximate prior knowledge of the plant. Section 6 is devoted to application of the proposed PI-PBC for port-Hamiltonian $(\mathrm{pH})$ systems [1] and a temperature regulation problem. The paper is wrapped-up with concluding remarks in Section 7. A preliminary version of this paper was reported in [14].

Notation $I_{n}$ is the $n \times n$ identity matrix and $\mathbf{0}_{n \times s}$ is an $n \times s$ matrix of zeros, $\mathbf{0}_{n}$ is an $n-$ dimensional column vector of zeros. Given $a_{i} \in \mathbb{R}, i \in \bar{n}:=\{1, \ldots, n\}$, we denote with $\operatorname{col}\left(a_{i}\right)$ the $n$-dimensional column vector and $\operatorname{diag}\left\{a_{i}\right\}$ the diagonal $n \times n$ matrix with elements $a_{i}$. For $x \in \mathbb{R}^{n},|x|$ is the Euclidean norm. For mappings of scalar argument $g: \mathbb{R} \rightarrow \mathbb{R}^{s}, g^{\prime}$ and $g^{\prime \prime}$ denote first and second order differentiation, respectively. For mappings $f: \mathbb{R}^{n} \rightarrow \mathbb{R}, \nabla f:=\left(\frac{\partial f}{\partial x}\right)^{\top}$ and $\nabla^{2} f:=\frac{\partial^{2} f}{\partial x^{2}}$. For the distinguished element $x^{*} \in \mathbb{R}^{n}$ and any mapping $F: \mathbb{R}^{n} \rightarrow \mathbb{R}^{s}$ we denote $F^{*}:=F\left(x^{*}\right)$ and the error signal $\tilde{F}(x):=F(x)-F^{*}$. 


\section{PROBLEM FORMULATION}

In this section we formulate the control problem addressed in the paper, enunciate the assumptions made on the plant to solve it and make some remarks on these assumptions.

\subsection{Robust PI control problem}

Consider the nonlinear, input affine, system

$$
\dot{x}=f(x)+G u,
$$

where $x \in \mathbb{R}^{n}, u \in \mathbb{R}^{m}, n>m, f: \mathbb{R}^{n} \rightarrow \mathbb{R}^{n}$ is an unknown smooth mapping, $G \in \mathbb{R}^{n \times m}$ is constant verifying $\operatorname{rank}(G)=m$.

The following is a key assumption made throughout the paper.

\section{Assumption 1}

The matrix $G$ has $n-m$ zero rows. Without lost of generality ${ }^{\dagger}$ it is assumed of the form

$$
G=\left[\begin{array}{c}
\mathbf{0}_{(n-m) \times m} \\
G_{2}
\end{array}\right],
$$

where $G_{2} \in \mathbb{R}^{m \times m}$ is known.

This assumption can be easily obviated introducing state and input changes of coordinates. Indeed, it is well-known-see, e.g., Theorem 2 of Section 2.7 of [15]-that for any full rank, matrix $G \in \mathbb{R}^{n \times m}$ there exists (elementary) full rank matrices $T \in \mathbb{R}^{n \times n}$ and $S \in \mathbb{R}^{m \times m}$ such that

$$
T G S=\left[\begin{array}{c}
\mathbf{0}_{(n-m) \times m} \\
I_{m}
\end{array}\right] .
$$

Consequently, introducing $z=T x$ and $v=S^{-1} u$ the system (1) takes the desired form

$$
\dot{z}=w(z)+\left[\begin{array}{c}
\mathbf{0}_{(n-m) \times m} \\
I_{m}
\end{array}\right] v,
$$

where $w(z)=T f\left(T^{-1} z\right)$. We should note, however, that a change of state representation destroysin general-the original structure of the system, whose knowledge may be critical for the verification of the second assumption below. This fact is clearly illustrated in the physical examples considered in Section 6. For this reason, we prefer to leave it as an standing assumption.

\footnotetext{
${ }^{\dagger}$ See R6 in the next subsection and Subsection 5.1 for more general forms of $G$.
} 
Motivated by Assumption 1 we find convenient to define a partition of the state vector into its un-actuated and actuated components as

$$
x=\left[\begin{array}{c}
x_{u} \\
x_{a}
\end{array}\right], x_{u}:=\left[\begin{array}{c}
x_{1} \\
x_{2} \\
\vdots \\
x_{n-m}
\end{array}\right], x_{a}:=\left[\begin{array}{c}
x_{n-m+1} \\
x_{n-m+2} \\
\vdots \\
x_{n}
\end{array}\right] .
$$

It is assumed that only $x_{a}$ is available for measurement.

The unforced system, that is, $\dot{x}=f(x)$, has a stable equilibrium at the origin with a partially known Lyapunov function. We are interested in controlling the system with a PI at a non-zero equilibrium-a situation that arises in most practical applications. Thus, we are given a desired equilibrium point, $x^{\star} \in \mathbb{R}^{n}$, and the control goal is to ensure stability of this equilibrium using a PI control law of the form

$$
\begin{gathered}
\dot{z}=-K_{I} \psi\left(x_{a}, x^{*}\right) \\
u=-K_{P} \psi\left(x_{a}, x^{*}\right)+z
\end{gathered}
$$

where $z \in \mathbb{R}^{m}$ is the controller state, $K_{P} \in \mathbb{R}^{m \times m}$ and $K_{I} \in \mathbb{R}^{m \times m}$ are tuning gains and $\psi$ : $\mathbb{R}^{m} \times \mathbb{R}^{n} \rightarrow \mathbb{R}^{m}$ is a mapping designed with the partial knowledge of the aforementioned Lyapunov function.

The following, practically reasonable, assumption is made throughout the paper.

\section{Assumption 2}

The desired equilibrium point $x^{\star}$ belongs to the assignable equilibrium set, that is,

$$
x^{\star} \in \mathcal{E}:=\left\{x \in \mathbb{R}^{n} \mid\left[\begin{array}{lll}
I_{n-m} & \mid \mathbf{0}_{(n-m) \times n}
\end{array}\right] f(x)=0\right\} .
$$

\subsection{Assumptions on the open-loop plant}

The following assumption identifies the class of vector fields $f(x)$ for which we provide an answer to the problem.

\section{Assumption 3}

For the system (1) there exists a twice-differentiable, positive definite function $H: \mathbb{R}^{n} \rightarrow \mathbb{R}_{\geq 0}$, verifying the following.

(i) $[\nabla H(x)]^{\top} f(x) \leq 0$.

(ii) $\left[\nabla H(x)-\nabla H\left(x^{\star}\right)\right]^{\top} \tilde{f}(x)=:-Q(x) \leq 0$.

(iii) The function $H(x)$ is of the form

$$
H(x)=H_{u}\left(x_{u}\right)+H_{a}\left(x_{a}\right)
$$


with

$$
H_{a}\left(x_{a}\right)=\sum_{i=n-m+1}^{n} d_{i} \phi_{i}\left(x_{i}\right),
$$

where the function $H_{u}: \mathbb{R}^{n-m} \rightarrow \mathbb{R}$ and the constants $d_{i}>0$ are unknown but the functions $\phi_{i}: \mathbb{R} \rightarrow \mathbb{R}$ are known.

(iv) The functions $H_{u}\left(x_{u}\right)$ and $\phi_{i}\left(x_{i}\right)$ are convex.

\subsection{Discussion}

The following remarks regarding the assumptions are in order.

R1 Although the set of assignable equilibria $\mathcal{E}$ is not known, it is reasonable to assume that we have enough prior knowledge about the plant to select the desired operating point as a feasible equilibrium. Hence, Assumption 2 is practically reasonable.

R2 A corollary of Assumption 2 is that the constant input $u^{\star}$, that assigns the equilibrium, is uniquely defined as

$$
u^{\star}:=\left(G_{2}^{\top} G_{2}\right)^{-1}\left[\mathbf{0}_{m \times(n-m)} \quad G_{2}^{\top}\right] f^{\star} .
$$

Notice that, without knowledge of $f(x)$, this constant cannot be computed.

R3 Since the open-loop system (1) has a stable equilibrium at the origin Assumption 3 (i) follows as a corollary of Lyapunov's converse theorems [16]. As will become clear below Assumption 3 (ii) and (iv) are required to prove passivity of the incremental model as done in [2].

R4 We underscore that no assumption, beyond twice differentiability and convexity, is imposed on the unknown component $H_{u}\left(x_{u}\right)$ of the Lyapunov function of the open-loop system $H(x)$. On the other hand, stricter conditions are imposed on the second component $H_{a}\left(x_{a}\right)$, with uncertainty captured by the unknown constants $d_{i}$.

R5 Assumptions 3 (iii) and Assumption 1 are the key requirements imposed on the plant to design the robust PI-PBC. This assumption is satisfied by a large class of physical systems, including the thermal systems studied in Section 6 and a class of port-Hamiltonian systems [1].

R6 It can be noticed that the class of port-Hamiltonian systems of the form:

$$
\dot{x}=(\mathcal{J}-\mathcal{R}) \nabla H(x)+G u
$$

with constant interconnection $\mathcal{J}=-\mathcal{J}^{\top}$ and damping $\mathcal{R}=\mathcal{R}^{\top} \geq 0$ matrices satisfies Assumption 3 (i) and (ii). Indeed, Assumption 3 (i) is satisfied since

$$
[\nabla H(x)]^{\top}(\mathcal{J}-\mathcal{R}) \nabla H(x)=-[\nabla H(x)]^{\top} \mathcal{R} \nabla H(x) \leq 0 .
$$

Similarly, Assumption 3 (ii) holds since

$$
\begin{aligned}
& {\left[\nabla H(x)-\nabla H\left(x^{*}\right)\right]^{\top}(\mathcal{J}-\mathcal{R})\left[\nabla H(x)-\nabla H\left(x^{*}\right)\right]=} \\
& -\left[\nabla H(x)-\nabla H\left(x^{*}\right)\right]^{\top} \mathcal{R}\left[\nabla H(x)-\nabla H\left(x^{*}\right)\right] \leq 0 .
\end{aligned}
$$


R7 Regarding Assumptions 1, in the more general case when $G$ is not of the form (2) an additional shuffling of the rows of $G$ is needed in the design. This procedure is explained in Subsection 5.1.

R8 For quadratic Lyapunov functions of the form $H(x)=x^{\top} P x$, with $P>0$, Assumption 3 (ii) is satisfied if the open-loop system is convergent in the sense of Demidovich [17]. That is, if it satisfies

$$
P \nabla f(x)+[\nabla f(x)]^{\top} P \leq 0 .
$$

\section{PRELIMINARY LEMMATA}

Unless otherwise indicated, throughout the rest of the paper Assumption 1 holds. Define for the system (1) the output

$$
y=G^{\top} \nabla H(x)=G_{2}^{\top} D \Phi\left(x_{a}\right),
$$

where

$$
\begin{aligned}
D:= & {\left[\begin{array}{cccc}
d_{n-m+1} & 0 & \ldots & 0 \\
0 & d_{n-m+2} & \ldots & 0 \\
\vdots & \vdots & \vdots & \vdots \\
0 & 0 & \ldots & d_{n}
\end{array}\right]>0 } \\
\Phi\left(x_{a}\right):= & {\left[\begin{array}{c}
\phi_{n-m+1}^{\prime}\left(x_{n-m+1}\right) \\
\vdots \\
\phi^{\prime}\left(x_{n}\right)
\end{array}\right] . }
\end{aligned}
$$

A corollary of the theorem of Hill and Moylan [1] is that, if Assumption 3 (i) holds, the system (1), (7) defines a passive mapping $u \mapsto y$ with storage function $H(x)$.

To operate the system at a non-zero equilibrium it is necessary to shift the minimum of the storage function and define the passivity property between the incremental input and the output error. Towards this end, we recall Proposition 1 of [2] and state it as a lemma below.

\section{Lemma 1}

Consider the incremental model of the system (1), (7)

$$
\begin{aligned}
\dot{x} & =f(x)+G u^{\star}+G \tilde{u}, \\
e & =G_{2}^{\top} D \tilde{\Phi}\left(x_{a}\right),
\end{aligned}
$$

where $\tilde{u}=u-u^{\star}$ is the incremental input. Under Assumptions 1-3 the mapping $\tilde{u} \mapsto e$ is passive with storage function $U: \mathbb{R}^{n} \rightarrow \mathbb{R}_{\geq 0}$ given by

$$
U(x)=H(x)-x_{u}^{\top} \nabla H_{u}^{*}-x_{a}^{\top} D \Phi^{\star}+k,
$$

where $k$ is a constant that ensures $U\left(x^{\star}\right)=0$. More precisely,

$$
\dot{U}=-Q(x)+e^{\top} \tilde{u},
$$


where $Q(x)$ is defined in Assumption 3 (ii).

One of the main interests of passive systems is that they can be globally stabilized with PI controls (with arbitrary positive definite gains). This well-known fact is stated in the lemma below, whose proof is given to streamline the presentation of our main result.

\section{Lemma 2}

Consider the system (1) verifying Assumptions 1-3 in closed-loop with the PI-PBC

$$
\begin{aligned}
e & =G_{2}^{\top} D \widetilde{\Phi}\left(x_{a}\right) \\
\dot{z} & =-K_{I} e \\
u & =-K_{P} e+z .
\end{aligned}
$$

For all positive definite gain matrices $K_{P} \in \mathbb{R}^{m \times m}$ and $K_{I} \in \mathbb{R}^{m \times m}$ all trajectories are bounded, the equilibrium point $(x, z)=\left(x^{*}, u^{*}\right)$ is globally stable (in the sense of Lyapunov) and the augmented error signal

$$
e_{a}:=\left[\begin{array}{c}
Q(x) \\
e
\end{array}\right]
$$

where $Q(x)$ is defined in Assumption 3 (ii), verifies

$$
\lim _{t \rightarrow \infty} e_{a}(t)=0
$$

Moreover, if $e_{a}$ is a detectable output for the closed-loop system then the equilibrium point is asymptotically stable.

\section{Proof}

Defining $\tilde{z}:=z-u^{*}$ the last two equations of the controller (11) may be written in the form

$$
\begin{aligned}
& \dot{\tilde{z}}=-K_{I} e \\
& \tilde{u}=-K_{P} e+\tilde{z} .
\end{aligned}
$$

Consider the Lyapunov function candidate

$$
W(\tilde{z}, x)=U(x)+\frac{1}{2} \tilde{z}^{\top} \Lambda_{I} \tilde{z},
$$

where $\Lambda_{I}>0$. The time derivative of the Lyapunov function along the trajectories of the closedloop system is

$$
\begin{aligned}
\dot{W} & =-Q(x)+e^{\top} \tilde{u}+\tilde{z}^{\top} \Lambda_{I} \dot{\tilde{z}} \\
& =-Q(x)-e^{\top} K_{P} e+\tilde{z}^{\top} e-\tilde{z}^{\top} \Lambda_{I} K_{I} e .
\end{aligned}
$$

Setting $\Lambda_{I}=K_{I}^{-1}$ yields

$$
\dot{W}=-Q(x)-e^{\top} K_{P} e .
$$

The proof is complete invoking standard Lyapunov arguments [16]. 


\section{THE ROBUST PI-PBC}

As indicated in R4 of Subsection 2.3 the matrix $D$ is unknown. Hence, the error signal $e$ cannot be constructed and the PI-PBC (11) is not implementable. This motivates our main result given below.

\section{Proposition 1}

Consider system (1) verifying Assumptions 1-3 in closed-loop with the robust PI-PBC

$$
\begin{aligned}
& u=-K_{P} \widetilde{\Phi}\left(x_{a}\right)+z \\
& \dot{z}=-K_{I} \widetilde{\Phi}\left(x_{a}\right),
\end{aligned}
$$

with the controller gains

$$
\begin{aligned}
K_{P} & =G_{2}^{-1} \Gamma_{P} \\
K_{I} & =G_{2}^{-1} \Gamma_{I} .
\end{aligned}
$$

For all diagonal, positive definite matrices $\Gamma_{P} \in{ }^{m \times m}$ and $\Gamma_{I} \in \mathbb{R}^{m \times m}$ we have the following.

(i) All trajectories are bounded and the equilibrium point $(x, z)=\left(x^{*}, u^{*}\right)$ is globally stable (in the sense of Lyapunov).

(ii) The augmented error signal $e_{a}$ defined in (12) verifies (13).

(iii) If $e_{a}$ is a detectable output for the closed-loop system then the equilibrium point is globally asymptotically stable.

\section{Proof}

Some simple manipulations prove that

$$
K_{P} \widetilde{\Phi}\left(x_{a}\right)=G_{2}^{-1} \Gamma_{P} D^{-1} \quad{ }_{2}^{-\top} G_{2}^{\top} D \widetilde{\Phi}\left(x_{a}\right)=\Lambda_{P} e,
$$

where we defined the matrix

$$
\Lambda_{P}:=G_{2}^{-1} \Gamma_{P} D^{-1} G_{2}^{-\top},
$$

and used the definition of $e$ in (11). Invoking Sylvester's Law of Inertia [15], and the fact that $\Gamma_{P}$ and $D$ are diagonal and positive definite, we have that $\Lambda_{P}>0$.

Next choose

$$
\Lambda_{I}:=G_{2}^{\top} \quad \Gamma_{I}^{-1} G_{2},
$$

that is, also, positive definite for all diagonal, positive definite $\Gamma_{I}$. Then

$$
\Lambda_{I} K_{I} \widetilde{\Phi}(x)=G_{2}^{\top} D \widetilde{\Phi}\left(x_{a}\right)=e .
$$

Replacing (19) and (22) in the controller equations yields

$$
\begin{aligned}
& \tilde{u}=-\Lambda_{P} e+\tilde{z} \\
& \dot{\tilde{z}}=-\Lambda_{I}^{-1} e .
\end{aligned}
$$


Consequently, the time derivative of the Lyapunov function (16) becomes now

$$
\dot{W}=-Q(x)-e^{\top} \Lambda_{P} e,
$$

completing the proof.

To obtain an implementable version of the robust PI-PBC it was necessary to carry-out two tasks. First, to make the damping injection, introduced by the proportional term, function of the unknown matrix $D$. Indeed, replacing (20) in (19) we get

$$
K_{P} \widetilde{\Phi}(x)=G_{2}^{-1} \Gamma_{P} D^{-1} G_{2}^{-\top} e .
$$

Second, make the gain $\Lambda_{I}$ of the Lyapunov function (15) also a function of $D$-see (21).

An important observation is that, even though the controller only requires measurement of the actuated terms of the state $x_{a}$, it achieves regulation of the full state vector.

\section{ADDITIONAL REMARKS ON THE PI-PBC}

In this section we explain how to proceed when $G$ is not of the form (2), discuss the reasons that stymie the use of adaptation and the inability to state a robustness result based on continuity and approximate prior knowledge of the matrix $D$.

\subsection{General $G$ (with $n-m$ zero rows)}

Instrumental to design the robust PI-PBC was the particular form of $H(x)$ defined in Assumption 3 (iii). In view of the construction of the robust PI-PBC, it is clear that if $G$ is not of the form (2) the assumption must be modified redefining the actuated and un-actuated coordinates.

To avoid cluttering the notation we will explain the procedure only for the case when $n=3$ and $m=2$-the general case follows verbatim. Assume, furthermore, that $G$ is of the form

$$
G=\left[\begin{array}{c}
g_{1}^{\top} \\
\mathbf{0}_{1 \times 2} \\
g_{3}^{\top}
\end{array}\right]
$$

The form of $H(x)$ given in Assumption 3 (iii) must be, accordingly, modified to

$$
H(x)=H_{u}\left(x_{2}\right)+d_{1} \phi\left(x_{1}\right)+d_{3} \phi\left(x_{3}\right) .
$$

In this case the passive output $e$ for the incremental model becomes

$$
G^{\top}\left[\nabla H(x)-\nabla H\left(x^{*}\right)\right]=G_{s}\left[\begin{array}{cc}
d_{1} & 0 \\
0 & d_{3}
\end{array}\right]\left[\begin{array}{c}
\widetilde{\Phi}_{1}\left(x_{1}\right) \\
\widetilde{\Phi}_{3}\left(x_{3}\right)
\end{array}\right] .
$$

where

$$
G_{s}:=\left[\begin{array}{lll}
g_{1} & \mid & g_{3}
\end{array}\right]
$$


The robust $\mathrm{PI}-\mathrm{PBC}$ is given by

$$
\begin{aligned}
& u=-G_{s}^{-1} \Gamma_{P}\left[\begin{array}{c}
\widetilde{\Phi}_{1}\left(x_{1}\right) \\
\widetilde{\Phi}_{3}\left(x_{3}\right)
\end{array}\right]+z \\
& \dot{z}=-G_{s}^{-1} \Gamma_{I}\left[\begin{array}{c}
\widetilde{\Phi}_{1}\left(x_{1}\right) \\
\widetilde{\Phi}_{3}\left(x_{3}\right)
\end{array}\right],
\end{aligned}
$$

where $\Gamma_{P}$ and $\Gamma_{I}$ are arbitrary, diagonal, positive definite matrices.

Before closing this subsection we remark that our construction critically relies on the assumption of existence of $n-m$ zero rows in $G$. Indeed, it is possible to show that if this is not the case, even assuming $H(x)$ of the form

$$
H(x)=\sum_{i=1}^{n} d_{i} \phi_{i}\left(x_{i}\right)
$$

it is not possible to find an $m \times m$ positive definite matrix $\Lambda$, which will depend on $D$, such that the matrix $\Lambda G^{\top} D$ is independent of $D$. The fact that this is not possible for all matrices $G$ is obvious considering the counterexample $G=\operatorname{col}(1,1)$. Hence, the assumption of existence of $n-m$ zero rows in $G$ is necessary to solve the problem.

\subsection{Difficulties for adaptation}

A natural alternative to the robust PI-PBC presented above is to assume a parametrisation of $f(x)$ and try to estimate this parameters or, in a direct approach, estimate the matrix $D$ that defines the passive output. The indirect approach, as is well-known, relies on parameter convergence that requires persistency of excitation-a property that is not satisfied in the regulation tasks where PI control is effective.

Let us see what are the difficulties for the application of a direct adaptation approach. Towards this end, we propose the adaptive PI-PBC

$$
\begin{aligned}
\dot{\hat{D}} & =F(x, z) \\
\hat{e} & =G_{2}^{\top} \hat{D} \widetilde{\Phi}\left(x_{a}\right) \\
\dot{z} & =-K_{I} \hat{e} \\
u & =-K_{P} \hat{e}+z,
\end{aligned}
$$

where the parameter adaptation law $F: \mathbb{R}^{n} \times \mathbb{R}^{m} \rightarrow \mathbb{R}^{m \times m}$ is to be defined. ${ }^{\ddagger}$ Defining $\tilde{e}:=\hat{e}-e$ the last two equations of the controller may be written in the form

$$
\begin{aligned}
& \dot{\tilde{z}}=-K_{I}(e+\tilde{e}) \\
& \tilde{u}=-K_{P}(e+\tilde{e})+\tilde{z} .
\end{aligned}
$$

\footnotetext{
${ }_{\ddagger}^{\ddagger}$ Notice that, in contrast to the robust PI-PBC, we have assumed that the full state is measurable.
} 
The time derivative of the Lyapunov function (15) with $\Lambda_{I}=K_{I}^{-1}$ is now

$$
\begin{aligned}
\dot{W} & =-Q(x)-\hat{e}^{\top} K_{P} \hat{e}-\tilde{u}^{\top} \tilde{e} \\
& =-Q(x)-\hat{e}^{\top} K_{P} \hat{e}-\tilde{u}^{\top} G_{2}^{\top} \tilde{D} \widetilde{\Phi}\left(x_{a}\right)
\end{aligned}
$$

where we underscore the presence of the last right hand term. If $\tilde{u}$ were known the standard procedure of augmenting the Lyapunov function with a term $\operatorname{trace}\left(\tilde{D}^{\top} \tilde{D}\right)$ and cancelling the signindefinite term with a suitable choice of $F(x, z)$ would do the job. Alas, $u^{*}$ is not known, hence this approach is not feasible.

Adding an adaptation for the constant $u^{*}$ is also not a trivial task, because of the bilinear nature of the joint estimation problem.

\subsection{Comments on robustness based on continuity}

The availability of a bona fide Lyapunov function for the known parameters PI-PBC, i.e., $W(x, \tilde{z})$, suggests that stability will be preserved if the matrix $D$ is replaced by a "good", constant estimate of it, say $D_{0}$. More precisely, it is expected that replacing the controller (11) by

$$
\begin{aligned}
e_{0} & =G_{2}^{\top} D_{0} \widetilde{\Phi}\left(x_{a}\right) \\
\dot{z} & =-K_{I} e_{0} \\
u & =-K_{P} e_{0}+z,
\end{aligned}
$$

where

$$
D=D_{0}+\Delta, \Delta:=\operatorname{diag}\left\{\delta_{i}\right\}
$$

would ensure stability if $\left|\operatorname{col}\left(\delta_{i}\right)\right|$ is sufficiently small. Unfortunately, since the Lyapunov function is not strict, this conjecture cannot be analytically validated. Indeed, in this case the time derivative of the Lyapunov function (15) with $\Lambda_{I}=K_{I}^{-1}$ is now

$$
\begin{aligned}
\dot{W} & =-Q(x)+e^{\top} \tilde{u}-\tilde{z}^{\top}\left(e-G_{2}^{\top} \Delta \widetilde{\Phi}\left(x_{a}\right)\right) \\
& =-Q(x)-e_{0}^{\top} K_{P} e_{0}-\left(K_{P} e_{0}-\tilde{z}\right)^{\top} G_{2}^{\top} \Delta \widetilde{\Phi}\left(x_{a}\right) .
\end{aligned}
$$

While the term $e_{0}^{\top} K_{P} G_{2}^{\top} \Delta \widetilde{\Phi}\left(x_{a}\right)$ can be dominated for "small" $\Delta$, there is no way we can dominate the remaining term $\tilde{z}^{\top} K_{I} G_{2}^{\top} \Delta \widetilde{\Phi}\left(x_{a}\right)$ and the Lyapunov analysis cannot be completed with standard techniques.

This unfortunate situation does not mean, of course, that a continuity result of this type cannot be established. It simply reveals our inability to do it with the tools used to analyze the ideal case.

\section{APPLICATION TO TEMPERATURE REGULATION}

In this subsection we design a robust PI-PBC for the temperature regulation of a class of thermal systems-the so-called, Rapid Thermal Processes (RTP). Attention is concentrated on the verification of Assumption 3. Hence, unless otherwise indicated, Assumption 1 is not imposed. 


\subsection{System Description}

Similarly to $[18,19]$ we consider the following model of Rapid Thermal Processes

$$
\dot{T}=A_{1}\left[\Psi(T)-\Psi\left(T_{\text {rad }}\right)\right]+A_{2}\left(T-T_{\text {conv }}\right)+G u
$$

where $T \in \mathbb{R}_{\geq 0}^{n}$ represents the vector of temperatures, $\Psi(T):=\operatorname{col}\left(T_{i}^{4}\right)$ and $T_{\text {rad }}, T_{\text {conv }} \in \mathbb{R}_{\geq 0}^{n}$ are, respectively, the vectors of temperatures related to the radiation heat emission from environment and the convection air flows. The constant matrices $A_{1}, A_{2} \in \mathbb{R}^{n \times n}$ are Hurwitz and contain the radiation and the convection heat transfer coefficients. Also, the entries of $G \in \mathbb{R}^{n \times m}$ correspond to the heat transfer coefficients of the heating elements. Finally, $u \in \mathbb{R}^{m}$ is the controlled power applied to the heating elements. Physically, considering matrix $G$ as (2) means that for $m$ heating elements there are $n-m$ measured points that are not directly heated by these elements.

In the model above, as in [19], it is considered that the plant is heated almost uniformly so that the contribution of energy from conduction is too small with respect to the radiation transfer. Hence, the conduction heat transfer is neglected. We also assume the steady environment conditions, i.e., the values $T_{\text {rad }}$ and $T_{\text {conv }}$ are constant.

To simplify the notation we re-write the system (24) in the form

$$
\dot{T}=A_{1} \Psi(T)+A_{2} T+E+G u
$$

where

$$
E:=-A_{1} \Psi\left(T_{\text {rad }}\right)-A_{2} T_{\text {conv }} .
$$

Unlike $A_{1}, A_{2}$ and $E$ that are highly uncertain, the input matrix $G$ - that is defined by the induced heat flow-can be precisely identified. The control objective is then to design a robust PI, i.e., that does not require the knowledge of the uncertain parameters, to regulate the process around some desired temperature, which is not equal to the open-loop equilibrium, but belongs to the assignable equilibrium set, that is,

$$
T^{\star} \in\left\{T \in \mathbb{R}_{\geq 0}^{n} \mid G^{\perp}\left[A_{1} \Psi(T)+A_{2} T+E\right]=0\right\},
$$

where $G^{\perp} \in \mathbb{R}^{(n-m) \times n}$ is a full-rank left-annihilator of $G$.

To place the problem in the context of Proposition 1 we first shift the equilibrium of the openloop system to the origin and then proceed to verify Assumption 3. For, we introduce the standard change of coordinates

$$
x=T-\bar{T}
$$

where $\bar{T}$ is the open-loop equilibrium that satisfies

$$
A_{1} \Psi(\bar{T})+A_{2} \bar{T}+E=0 .
$$

Thus, the system (24) in the new coordinates takes the form (1) with

$$
f(x):=A_{1} \Psi(x+\bar{T})+A_{2}(x+\bar{T})+E,
$$


Associated to the desired temperature $T^{\star}$ we define the equilibrium to be stabilised

$$
x^{\star}:=T^{\star}-\bar{T} .
$$

\subsection{Passivity of the thermal system.}

The lemma below identifies conditions under which the system (24) satisfies Assumption 3 without imposing Assumption 1, that is, avoiding the partition of the coordinates into actuated and unactuated. Towards this end, the following assumption is needed.

\section{Assumption 4}

The matrix $A_{1}$ is diagonally stable [20]. That is, there exists $P \in \mathbb{R}^{n \times n}, P=\operatorname{diag}\left\{p_{i}\right\}>0$ such that

$$
P A_{1}+A_{1}^{\top} P=:-2 S<0
$$

Moreover, the matrix $A_{2}$ verifies

$$
A_{2}^{\top} P \operatorname{diag}\left\{T_{i}^{3}\right\}+\operatorname{diag}\left\{T_{i}^{3}\right\} P A_{2} \leq 0 .
$$

Conditions for diagonal stability of a matrix have been studied intensively, see [20] for a survey. Necessary and sufficient conditions were first reported in [21]— see also [22] for a simpler proof. For a Hurwitz matrix, a sufficient condition given in [23] is that it is a Metzler matrix (namely, its non diagonal elements are nonnegative). Note that due to physical nature of RTP systems the matrix $A_{1}$ usually belongs to this class.

Since $A_{2}$ is Hurwitz and $T_{i} \geq 0$, condition (31) is trivially satisfied if $A_{2}$ is diagonal, which is assumed in RTP models [19, 24].

Lemma 3

If Assumption 4 holds the vector field (28) satisfies Assumption 3 with

$$
H(x)=\sum_{i=1}^{n} p_{i} \phi_{i}\left(x_{i}\right)+k
$$

where

$$
\phi_{i}\left(x_{i}\right)=\frac{1}{5}\left(x_{i}+\bar{T}_{i}\right)^{5}-\Psi_{i}(\bar{T}) x_{i} .
$$

and

$$
k=-\frac{1}{5} \sum_{i=1}^{n} p_{i} \bar{T}_{i}^{5} .
$$

\section{Proof}

Point (iii) of Assumption 3 is trivially satisfied by (32).

We proceed now to prove point (i). Replacing (33) in (32) and grouping terms yields

$$
H(x)=\frac{1}{5} \sum_{i=1}^{n} p_{i}\left(x_{i}+\bar{T}_{i}\right)^{5}-x^{\top} P \Psi(\bar{T})+k,
$$


Now, notice that

$$
\nabla H(x)=P \Phi(x)
$$

where

$$
\Phi(x):=\Psi(x+\bar{T})-\Psi(\bar{T}) .
$$

On the other hand, from (27) it follows that the systems vector field may be written as

$$
f(x)=A_{1} \Phi(x)+A_{2} x .
$$

Consequently,

$$
\begin{aligned}
{[\nabla H(x)]^{\top} f(x, \theta) } & =\Phi^{\top}(x) P\left[A_{1} \Phi(x)+A_{2} x\right] \\
& =-\Phi^{\top}(x) S \Phi(x)+\Phi^{\top}(x) P A_{2} x
\end{aligned}
$$

where we have used (30) to obtain the second identity. Now, condition (31) ensures that the function $h: \mathbb{R}^{n} \rightarrow \mathbb{R}^{n}$

$$
h(x):=A_{2}^{\top} P \Psi(x),
$$

is monotonically decreasing [17]. That is, for all $a, b \in \mathbb{R}^{n}$,

$$
[h(a)-h(b)]^{\top}(a-b) \leq 0 .
$$

Consequently,

$$
\Phi^{\top}(x) P A_{2} x=[h(x+\bar{T})-h(\bar{T})]^{\top} x \leq 0
$$

completing the proof of point (i).

To prove point (ii) we notice that

$$
\tilde{f}(x)=A_{1} \tilde{\Phi}(x)+A_{2} \tilde{x}
$$

while

$$
\nabla H(x)-\nabla H\left(x^{*}\right)=P \tilde{\Phi}(x) .
$$

Hence, the claim is established invoking the same arguments used above and defining

$$
Q(x)=\tilde{\Phi}^{\top}(x) S \tilde{\Phi}(x) .
$$

Finally, the second derivative of the functions $\phi_{i}\left(x_{i}\right)$ yields

$$
\phi_{i}^{\prime \prime}\left(x_{i}\right)=4\left(x_{i}+\bar{T}_{i}\right)^{3}=4 T_{i}^{3},
$$

which is non-negative because $T_{i} \geq 0$. Hence, the functions $\phi_{i}\left(x_{i}\right)$ are convex as requested by condition (iv) of Assumption 3. This completes the proof.

Direct application of Lemma 1 leads to the following. 


\section{Corollary 1}

If Assumption 4 holds, the thermal system (24) defines a passive map $\tilde{u} \mapsto e$ with storage function $U(x)$, where

$$
\begin{aligned}
e & =G^{\top} P \tilde{\Phi}(x) \\
U(x) & =H(x)-x^{\top} P \Phi\left(x^{*}\right)-H\left(x^{*}\right)+\left(x^{*}\right)^{\top} P \Phi\left(x^{*}\right)
\end{aligned}
$$

\subsection{Robust PI-PBC of the thermal system}

To present the robust PI-PBC for systems verifying Assumption 1 we partition the vector of temperatures into its un-actuated and actuated components

$$
T=\left[\begin{array}{c}
T_{u} \\
T_{a}
\end{array}\right], T_{u}:=\left[\begin{array}{c}
T_{1} \\
T_{2} \\
\vdots \\
T_{n-m}
\end{array}\right], T_{a}:=\left[\begin{array}{c}
T_{n-m+1} \\
T_{n-m+2} \\
\vdots \\
T_{n}
\end{array}\right],
$$

partition $P$ as

$$
P=\left[\begin{array}{cc}
P_{1} & \mathbf{0}_{(n-m) \times m} \\
\mathbf{0}_{m \times(n-m)} & D
\end{array}\right],
$$

and do the same with the vector function $\Psi(T)$.

The following proposition is a consequence of Lemma 3 and Proposition 1.

\section{Proposition 2}

Consider the system (24) verifying Assumptions 1 and 4. Fix any desired temperature $T^{*}$ verifying (26) and define the PI-PBC

$$
\begin{aligned}
& u=-K_{P} \tilde{\Psi}_{a}\left(T_{a}\right)+z \\
& \dot{z}=-K_{I} \tilde{\Psi}_{a}\left(T_{a}\right),
\end{aligned}
$$

and the controller gains $K_{P}$ and $K_{I}$ are given by (18). For all diagonal, positive definite matrices $\Gamma_{P} \in \mathbb{R}^{m \times m}$ and $\Gamma_{I} \in \mathbb{R}^{m \times m}$ all trajectories are bounded and the equilibrium point $(T, z)=$ $\left(T^{*}, u^{*}\right)$ is globally asymptotically stable.

Proof

The proof of stability is established invoking item (i) of Proposition 1 and identifying

$$
\left.\tilde{\Phi}_{a}\left(x_{a}\right)\right|_{x_{a}=T_{a}-\bar{T}_{a}}=\tilde{\Psi}_{a}\left(T_{a}\right) .
$$

To prove asymptotic stability we invoke item (ii) and observe that the augmented error signal (12) is given in this case by

$$
e_{a}=\left[\begin{array}{c}
\tilde{\Psi}^{\top}(T) S \\
G_{2}^{\top} D
\end{array}\right] \tilde{\Psi}(T) .
$$

Since $e_{a}$ verifies (13) and $S$ is positive definite we conclude that $\tilde{\Psi}(T(t)) \rightarrow 0$ and consequently $T(t) \rightarrow T^{\star}$. 

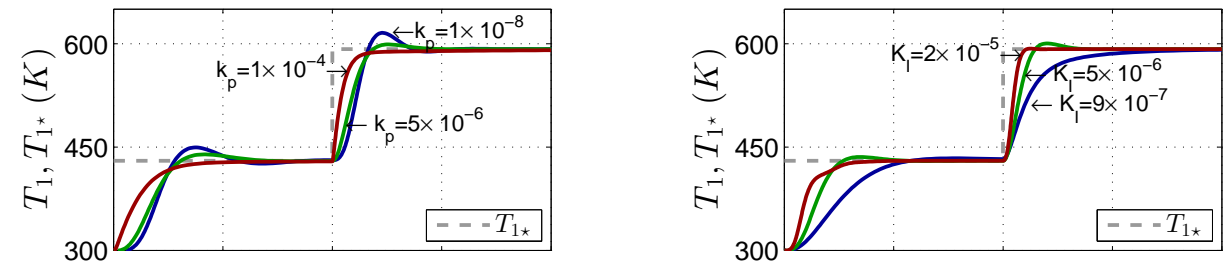

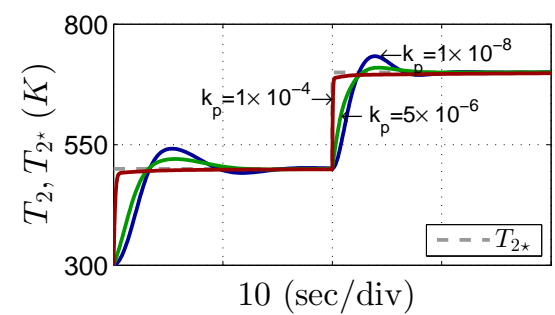

(a)

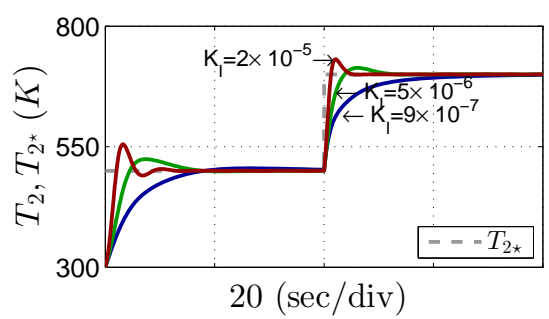

(b)

Figure 1. Simulation Result showing the system response : (a) For different gains $K_{p}$ letting $K_{I}=3 \times 10^{-6}$. (b) For different gains $K_{I}$ letting $K_{p}=6 \times 10^{-6}$.

\subsection{Numerical Simulation:}

Consider the thermal system (25) with

$$
A_{1}=\left[\begin{array}{cc}
-a_{11} & a_{12} \\
a_{21} & -a_{22}
\end{array}\right], A_{2}=\left[\begin{array}{cc}
-\alpha_{1} & 0 \\
0 & -\alpha_{2}
\end{array}\right], G=\left[\begin{array}{l}
0 \\
g
\end{array}\right], C=\left[\begin{array}{l}
c_{1} \\
c_{2}
\end{array}\right]
$$

where $a_{i j} \geq 0, \alpha_{i} \geq 0$. Notice that the system satisfies Assumption 4. Then, the assignable equilibria set is

$$
\mathcal{E}=\left\{T: T_{2} \in \mathbb{R}_{+},-a_{11} T_{1}^{4}+a_{12} T_{2}^{4}-\alpha_{1} T_{1}+c_{1}=0\right\}
$$

From Proposition 2, the controller

$$
\begin{aligned}
& \dot{z}=-K_{I}\left(T_{2}^{4}-\left(T_{2}^{\star}\right)^{4}\right) \\
& u=-K_{P}\left(T_{2}^{4}-\left(T_{2}^{\star}\right)^{4}\right)+z
\end{aligned}
$$

where $K_{p}=\frac{1}{g} \Gamma_{p}, K_{I}=\frac{1}{g} \Gamma_{I}$ and $\Gamma_{P}, \Gamma_{I} \in \mathbb{R}_{+}$asymptotically stabilizes the system at $T=T^{\star}$. The parameter values used in the simulation where: $a_{11}=1 \times 10^{-9}, a_{12}=\frac{1}{2} \times \times 10^{-9}, a_{21}=$ $1 \times 10^{-9}, a_{22}=1 \times 10^{-9}, \alpha_{1}=1 \times 10^{-4}, \alpha_{2}=\frac{1}{2} \times 10^{-4}, g=1, c_{1}=3, c_{2}=1.7, \Gamma_{p}=$ $8 \times 10^{-5}$ and $\Gamma_{I}=1 \times 10^{-5}$. In the simulation, the control objective is initially fixed at $T_{2 \star}=500 \mathrm{~K}$, then it is suddenly changed to $T_{2 \star}=700 \mathrm{~K}$. From (35), the corresponding values for $T_{1 \star}$ are, respectively, $430.06 \mathrm{~K}$ and $592.20 \mathrm{~K}$. Fig. 1 shows the simulation results. In Fig. 1a the response of the system when varying control parameter $K_{p}$ and letting $K_{I}=3 \times 10^{-6}$ is depicted. As it can be noticed from the same figure, the larger is the value in $K_{p}$ the faster is the convergence. In Fig. $1 \mathrm{~b}$ it is shown the response of the system when $K_{I}$ is varying while $K_{P}=6 \times 10^{-6}$. From the figure, it can be seen that large values in $K_{I}$ causes overshoots in the response of $T_{2}$. 


\section{CONCLUDING REMARKS}

In this work we identify a class of nonlinear systems for which it is possible to design robust PI controllers with guaranteed stability properties (see also [14]). The class consists of input affine systems with known, constant input matrix $G$ and $n-m$ zero rows. We assume that only the states associated to the non-zero rows of $G$ are measurable.The systems have an open-loop stable equilibrium, but is different from the desired operating point. To handle this situation, we follow [2] and generate new passive outputs for the incremental model, hence the name PI-PBC. Associated to the open-loop stable equilibrium a Lyapunov function of the form (4) is assumed to exist. We underscore that, besides convexity, there is no assumption on the function $H_{u}\left(x_{u}\right)$, which is unknown. Moreover, the controller does not require the measurement of $x_{u}$. The functions $\phi_{i}\left(x_{i}\right)$ are assumed convex and known, but the coefficient $d_{i}$ are unknown. Under these conditions, we show that, for a well identified class of PI tuning gains, see (18), global stability of the proposed $\mathrm{PI}-\mathrm{PBC}$ is guaranteed. Conditions that ensure global asymptotic stability, are also derived.

\section{REFERENCES}

1. van der Schaft A. $L_{2}$-Gain and Passivity Techniques in Nonlinear Control. Springer-Verlag New York, Inc., 1996.

2. Jayawardhana B, Ortega R, García-Canseco E, Castaños F. Passivity of nonlinear incremental systems: Application to PI stabilization of nonlinear RLC circuits. Systems \& Control Letters 2007; 56(9-10):618-622.

3. Castaños F, Jayawardhana B, Ortega R, García-Canseco E. Proportional plus integral control for set-point regulation of a class of nonlinear RLC circuits. Circuits, Systems \& Signal Processing 2009; 28(4):609-623.

4. Hernandez-Gomez M, Ortega R, Lamnabhi-Lagarrigue F, Escobar G. Adaptive PI stabilization of switched power converters. Control Systems Technology, IEEE Transactions on May 2010; 18(3):688-698.

5. Talj RJ, Ortega R, Hilairet M. A controller tuning methodology for the air supply system of a PEM fuel-cell system with guaranteed stability properties. International Journal of Control 2009; 61:1706-1719.

6. Marmidis G, Alexandridis A. A passivity-based PI control design for DC-drives. Control and Automation, 2009. MED '09. 17th Mediterranean Conference on, 2009; 1511-1516.

7. Meza JL, Santibañez V. Analysis via passivity theory of a class of nonlinear PID global regulators for robot manipulators. IASTED international conference on robotics and applications, 1999.

8. Donaire A, Junco S. On the addition of integral action to port-controlled Hamiltonian systems. Automatica 2009; 45(8): 1910 - 1916.

9. Ortega R, Romero JG. Robust integral control of port-Hamiltonian systems: The case of non-passive outputs with unmatched disturbances. Systems \& Control Letters 2012; 61(1):11 - 17.

10. Romero JG, Donaire A, Ortega R. Robust energy shaping control of mechanical systems. Systems \& Control Letters 2013; 62(9):770 - 780.

11. Antonelli R, Astolfi A. Continuous stirred tank reactors: Easy to stabilise? Automatica 2003; 39(10):1817 - 1827.

12. Ortega R, Kelly R. PID Self-Tuners: Some theoretical and practical aspects. Industrial Electronics, IEEE Transactions on Nov 1984; IE-31(4):332-338.

13. Sastry S, Bodson M. Adaptive Control: Stability, Convergence, and Robustness. Prentice Hall, 1994.

14. Aranovskiy S, Ortega R, Cisneros R. Robust PI Passivity-based control of nonlinear systems: Application to portHamiltonian systems and temperature regulation (Accepted). American Control Conference (ACC), 2014.

15. Lancaster P, Tismenetsky M. The Theory of Matrices. Academic Press, 1985.

16. Khalil HK. Nonlinear Systems. Prentice Hall, 2002.

17. Pavlov A, Pogromsky A, van de Wouw N, Nijmeijer H. Convergent dynamics, a tribute to Boris Pavlovich Demidovich. Systems \& Control Letters 2004; 52(34):257 - 261.

18. Ebert J, De Roover D, Porter L, Lisiewicz VA, Ghosal S, Kosut R, Emami-Naeini A. Model-based control of rapid thermal processing for semiconductor wafers. American Control Conference, 2004. Proceedings of the 2004, vol. 5, 2004; 3910-3921.

19. Schaper C, Cho Y, Kailath T. Low-order modeling and dynamic characterization of rapid thermal processing. Applied Physics A 1992; 54(4). 
20. Kaszkurewicz E, Bhaya A. Matrix Diagonal Stability in Systems and Computation. Birkhauser, 1991.

21. Barker GP, Berman A, Plemmons RJ. Positive diagonal solutions to the Lyapunov equations. Linear and Multilinear Algebra 1978; 5(4):249-256.

22. Shorten R, Mason O, King C. An alternative proof of the Barker, Berman, Plemmons (BBP) result on diagonal stability and extensions. Linear Algebra and its Applications 2009; 430(1):34- 40.

23. Farina L, Rinaldi S. Positive Linear Systems: Theory and Applications. John Wiley \& Sons, Inc, 2000.

24. Schaper C, Cho Y, Gyugyi P, Hoffmann G, Norman S, Park P, Boyd S, Franklin G, Kailath T, Saraswat K. Dynamics and Control of a Rapid Thermal Multiprocessor. Proceedings SPIE Conference on Rapid Thermal and Integrated Processing, 1992; 2-17. 